\title{
Development and validation of novel HPLC method for the estimation of Rutin in crude hydromethanolic leaf extract of Prosopis cineraria
}

\author{
Ram Singh Bishnoi*, Manish Kumar, Ajay Kumar Shukla, C.P. Jain \\ Department of Pharmaceutical Sciences, Mohanlal Sukhadia University, Udaipur, India
}

\begin{abstract}
A simple, specific, accurate and precise high performance liquid chromatography method has developed for the estimation of rutin in Prosopis cineraria. The chromatographic separation was achieved by using $\mathrm{C}_{18}$ column, $150 \times 4.6 \mathrm{~mm}$ i.d., $5 \mu$ bonded phase octadecylsilane (Thermo Labs Hypersil). Mobile phase was composed of 80 parts of methanol \& 20 parts of $0.05 \%$ formic acid. The $\mathrm{pH}$ of the mobile phase was 3.2.The retention time of rutin was found $5.7 \mathrm{~min}$ with $1 \mathrm{~mL} / \mathrm{min}$ flow rate at ambient temperature. The estimation was performed on PDA detector at $281 \mathrm{~nm}$. In this study, an excellent linearity was obtained with $\mathrm{r}^{2} 0.999$. Besides, the chromatographic peak was found sharp \& symmetric. The proposed method was validated in terms of the analytical parameters such as accuracy, linearity, precision, robustness, limit of detection (LOD), limit of quantification (LOQ) were determined based on the International Conference on Harmonization (ICH) guidelines. The detector response was linear in the range of $2-10 \mu \mathrm{g} / \mathrm{mL}$. The proposed method was successfully applied for the estimation of the constituents in crude extract of Prosopis cineraria. This study established a quantitative method for the determination of rutin from Prosopis cineraria.
\end{abstract}

Keywords: Prosopis cineraria, HPLC, Validation, Rutin.

Article Info: Received 27 Sep 2018; Review Completed 25 Oct 2018; Accepted 27 Oct 2018; Available online 15 Nov 2018

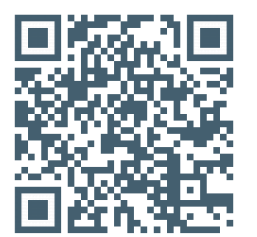

Cite this article as:

Bishnoi RS, Kumar M, Shukla AK, Jain CP, Development and validation of novel HPLC method for the estimation of Rutin in crude hydromethanolic leaf extract of Prosopis cineraria, Journal of Drug Delivery and Therapeutics. 2018; 8(6):68-73 DOI: http://dx.doi.org/10.22270/jddt.v8i6.2016

Ram Singh Bishnoi, Research Scholar, Department of Pharmaceutical Sciences, Mohanlal Sukhadia University, Udaipur-313001, Rajasthan, India

\section{INTRODUCTION}

The plant Prosopis cineraria (Khejri) is a flowering tree in the leguminous family Fabaceae. It is a small to medium size thorny, irregularly branched flowering tree, found widely in the Thar desert of Rajasthan, India and plays a vital role in preserving the ecosystem ${ }^{1}$.

It is one of the most important natural resources of arid regions of India because of its economic values (fuel, fodder), ecological role in preventing soil erosion. Prosopis cineraria have also been used in indigenous system of medicine as a folk medicine for various ailments. The bark is dry, acrid, bitter, with sharp taste; cooling anthelmintic, tonic; cures leprosy dysentery, asthma, leucoderma, piles, tremors of the muscle, wandering of the mind. The flowers are grounded mixed with sugar and used during pregnancy as safeguard against miscarriage. The ashes of bark are rubbed over the skin to remove hair. The smoke of the leaves is good for eye troubles. Fresh Leaves juice mixed with lemon juice is used for dyspepsia; extract of crushed pods is used for earache, toothache, pain relief from fractured bones. Aqueous extract of bark and leaves applied externally to treat skin disease disinfects wounds and promotes healinge-3.

Prosopis cineraria is mostly used as a folk medicine due to the presence numerous phytoconstituents like alkaloids (Spicigerine, Prosophylline), steroids (Campesterol, stigmasterol, sitosterol), tannins, phenolic compound (Gallic acid), flavone derivatives (Prosogerin A, B, C, D, and $\mathrm{E})$, etc has been isolated. In this respect, polyphenolic compounds, like flavonoids and phenolic acids, commonly found in plants have been reported to have multiple biological effects, including antioxidant and anti inflammatory activity. Synthetic antioxidants have toxicity, Thus interest in natural antioxidant, especially of plant origin, has greatly increased in recent years ${ }^{3}$.

Rutin (rutoside, quercetin-3-0-rutinoside) is 2-(3,4dihydroxyphenyl)-5,7-dihydroxy-3-[_-L-rhamnopyranosyl(1_6)-_-D-glucopyranosyloxy]-4H-chromen-4-one and is classified as a polyphenolic flavanoid (Fig. 1). It occurs in many food products of plant origin. Rutin appears as an odorless yellow crystalline powder that is practically insoluble in $\mathrm{H}_{2} \mathrm{O}$ and poorly soluble in alcohol ${ }^{4-7}$. Living 
organisms are incapable of synthesizing rutin. Therefore, it can only be ingested with plant products. Rutin and its aglycones were observed in various fruits, vegetables, tea leaves, coffee grains, etc ${ }^{8-9}$.

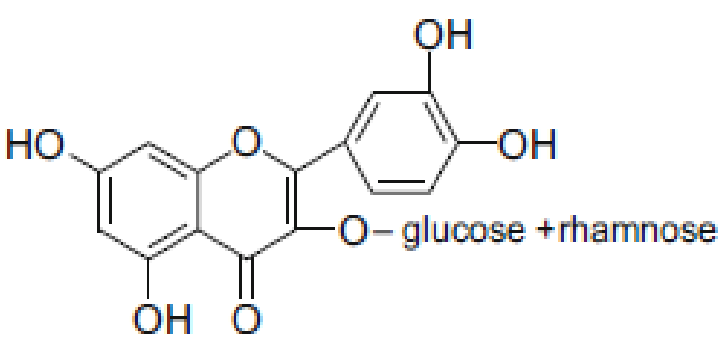

Figure 1: Structure of rutin

Identification of major and unique compounds in herbs as markers and development of analytical methodologies for monitoring them are the key steps involved in markerbased standardization ${ }^{10}$. High performance layer chromatography (HPLC) has recently emerged as a preferred analytical tool for fingerprints and quantification of marker compounds in herbal drugs because of its simplicity, sensitivity, accuracy, suitability for high throughput screening. HPLC method is a suitable method for estimation of chemical constituents present in plant materials ${ }^{11-14}$. The proposed method is optimized and validated as per the International Conference on Harmonization (ICH) guidelines ${ }^{15}$.

The literature survey showed that there was no report about the estimation of rutin constituent in leaf extract of Prosopis cineraria. Quantitative estimation of this compound is important for current research and a variety of methods are required for this and in the present study the quantification of rutin was done by HPLC method. A sensitive, accurate and specific HPLC method was developed and validated for the estimation of rutin in the hydro alcoholic and methanolic leaf extract of Prosopis cineraria.

\section{MATERIALS AND METHODS}

\subsection{Materials and reagents}

Rutin (98\%) was purchased from Sigma-Aldrich, Bangalore, India. Methanol and acetonitrile were of HPLC grade from Qualigens fine chemicals, Mumbai, India. All the reagents and chemicals used were of analytical and HPLC grade. Water (HPLC grade) was obtained from Milli Q RO system.

\subsection{Plant Material}

Fresh leaves of Prosopis cineraria were collected in month of November from Artiya Khurd village of Jodhpur
Rajasthan. A voucher specimen (JJ No. 847889) was submitted in the herbarium of Botanical Survey of India Jodhpur for authentication. BSI issued a certificate of authentication with ref no. BSI/AZRC/1.12014/Tech/ 2016-17-(Pl. Id)/1062. Leaves were collected in bulk, washed, shade dried \& coarsely powdered. Defated with petroleum ether \& macerated $70 \%$ methanol, the filtrated extract was then concentrated by using rotary evaporator and stored at $4^{\circ} \mathrm{C}$ prior to use.

\subsection{Preparation of standard solution}

The standard stock solution of $1000 \mu \mathrm{g} / \mathrm{mL}$ rutin was prepared by $70 \%$ methanol. This stock solution was stored in light resistant containers. The dilute standard solutions of concentration $2-10 \mu \mathrm{g} / \mathrm{mL}$ of rutin were prepared from above stock solution and used for calibration curve of rutin.

\subsection{Preparation of sample solution}

About $30 \mathrm{~g}$ of the powdered sample was weighed and defatted with the selected solvents by Soxhlet apparatus for $72 \mathrm{~h}$. The defatted residue was dried \& macerated with $70 \%$ methanol; the filtrate was dried at $50^{\circ} \mathrm{C}$ under reduced pressure in a rotary evaporator (Heidolph instrument $\mathrm{GmbH} \&$ Co.kg, Germany). The sample solution of extract of $1000 \mu \mathrm{g} / \mathrm{mL}$ was prepared by $70 \%$ methanol. After filtering through $0.45 \mu \mathrm{m}$ nylon filter paper, the extract was diluted with mobile phase injected directly.

\subsection{Instrumentation and Chromatographic conditions}

The estimation of rutin was performed on a Shimadzu liquid chromatographic system equipped with LC-10AT VP solvent delivery system (pumps), SPD M-10A VP photodiode array detector and Rheodyne 7725i injector with $20 \mu \mathrm{l}$ loop volume, LC Solution for data collection and processing (Shimadzu technologies, Japan). The mobile phase, MeOH: 0.05\% Formic acid pH $3.2(80: 20 \mathrm{v} / \mathrm{v})$ was pumped with a flow rate of $1 \mathrm{~mL} / \mathrm{min}$. The elution was monitored at $281 \mathrm{~nm}$. Peak identity was confirmed by retention time and spectrum comparison. All the analysis was performed at ambient temperature.

\section{RESULTS AND DISCUSSION}

\subsection{Method development and validation}

Upon application of the developed method, single specific peak was obtained for rutin (Figure 2). Rutin was identified in Prosopis cineraria hydro-methanolic extract. The quantitative analysis revealed that $(86.40+0.30 \mu \mathrm{g} / \mathrm{mg})$ rutin in the hydro-methanolic (70\% Methanol) extract. For validation of analytical methods, the guidelines of the International Conference on the Harmonization have recommended the accomplishment of linearity, accuracy tests, precision, detection and quantitation limit and robustness of the method. 


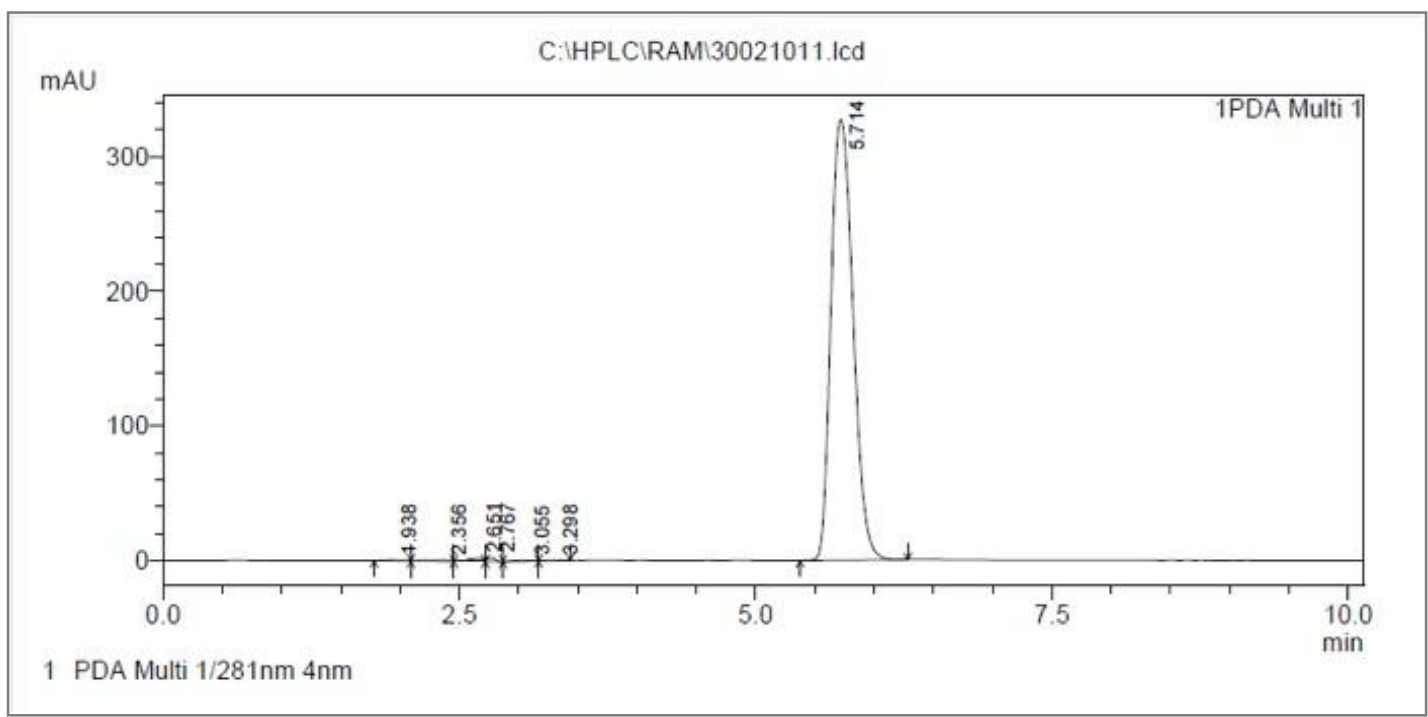

Figure 2: HPLC Chromatogram of standard Rutin

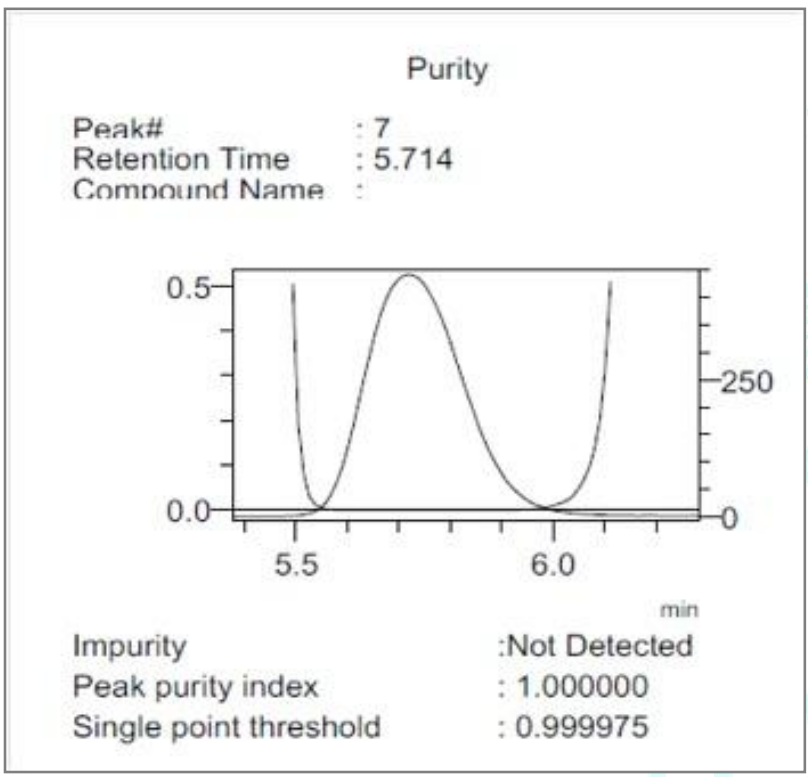

Figure 3: Peak purity of Rutin

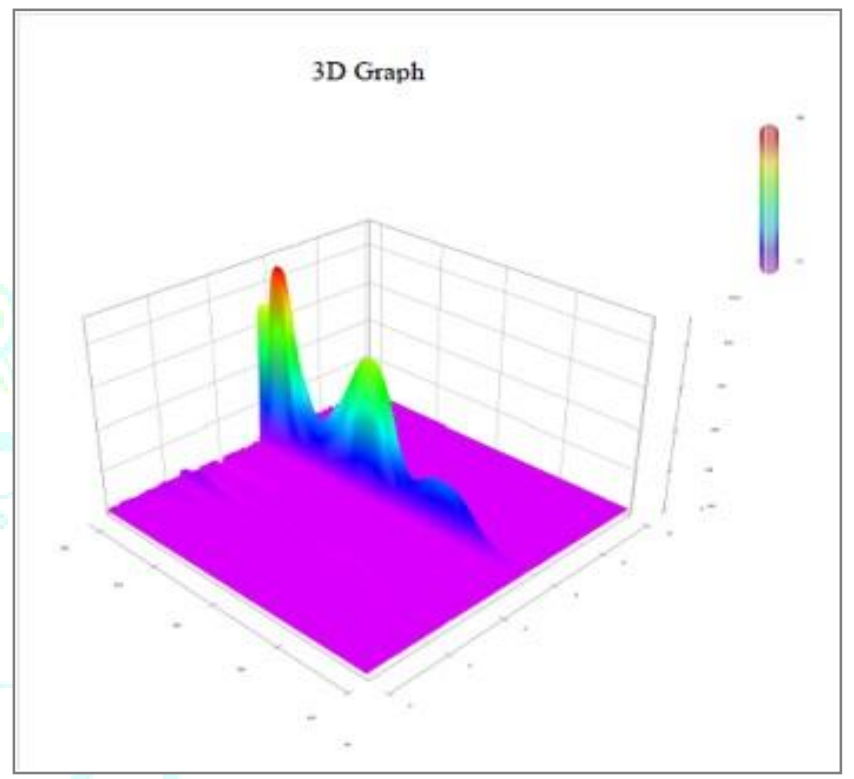

Figure 4: 3D-HPLC Chromatogram of standard Rutin

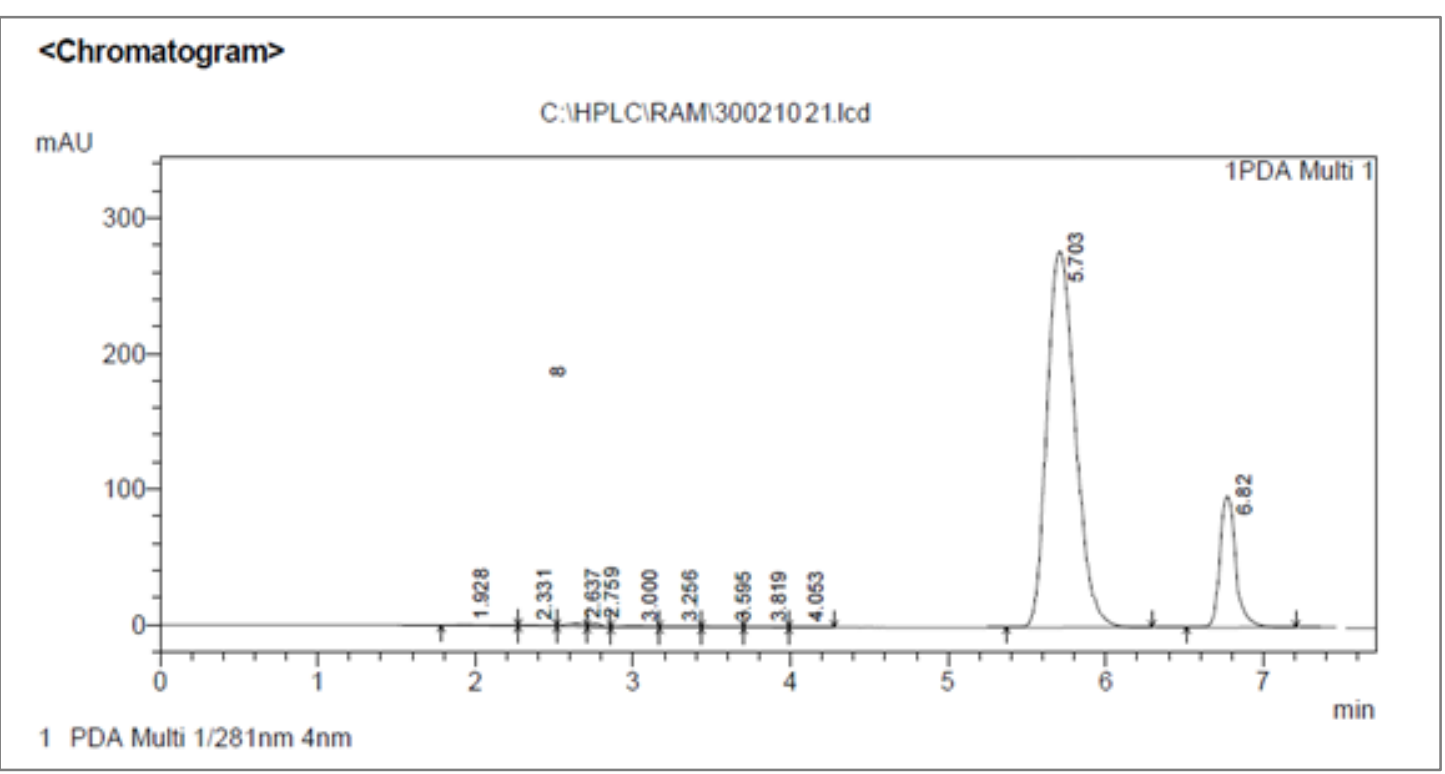

Figure 5: HPLC Chromatogram of crude hydro-methanolic extract of Prosopis cineraria 


\subsection{Linearity and range of the developed method}

For linearity study, five solutions in the range of 2-10 $\mu \mathrm{g} / \mathrm{mL}$ for rutin were analyzed. Each concentration was made and analyzed in triplicate. The peak areas obtained against each concentration of the analytes were used to build a linear regression equation and to determine value of correlation coefficient (Table 1). Good linearity was observed over the above-mentioned range with linear regression equation $\mathrm{Y}=423462 \mathrm{x}$ for rutin $(\mathrm{x}$ is concentration of analytes in $\mu \mathrm{g} / \mathrm{mL}$ and $\mathrm{Y}$ is peak area). The value of correlation coefficient was found to be 0.999 for rutin. The results indicate that the method is linear over the concentration range studied.

Table 1: Linearity data of Rutin

\begin{tabular}{|c|c|c|c|c|c|c|}
\hline \multirow{2}{*}{$\begin{array}{c}\text { Conc. } \\
(\mu \mathrm{g} / \mathrm{mL})\end{array}$} & \multicolumn{3}{|c|}{ Area under curve (AUC) Replicates } & \multirow[b]{2}{*}{ Mean } & \multirow[b]{2}{*}{ S.D. } & \multirow[b]{2}{*}{ R.S.D (\%) } \\
\hline & Rep-1 & Rep-2 & Rep-3 & & & \\
\hline 2 & 849193 & 849893 & 849493 & 849526.33 & 351.1884 & 0.04133 \\
\hline 4 & 1647586 & 1647086 & 1647044 & 1647238.67 & 301.5316 & 0.01830 \\
\hline 6 & 2553479 & 2550690 & 25532 & 2552479.00 & 1732.050 & 0.06785 \\
\hline 8 & & & 3392572 & 3392905.33 & 1527.525 & 0.04502 \\
\hline 10 & 4239465 & 4243588 & 4243140 & 4242064.33 & 2262.206 & 05332 \\
\hline
\end{tabular}

\subsection{Accuracy of the developed method}

This study was performed by adding known amounts of rutin to the all dilution of linearity $(2-10 \mu \mathrm{g} / \mathrm{mL})$ in three replicates. The recovery range for rutin was found to be 99.67 to $99.81 \%$. The \% relative standard deviation was found $0.0539 \%$ for rutin (Table 2 ).

Table 2: Recovery and accuracy data

\begin{tabular}{|c|c|c|c|}
\hline S. No. & Concentration $(\boldsymbol{\mu g} / \mathbf{m L})$ & Mean AUC & Response ratio \\
\hline 1 & 2 & 1695659 & 846755 \\
\hline 2 & 4 & 2543560 & 846927 \\
\hline 3 & 6 & 3391576 & 847034 \\
\hline 4 & 8 & 4239365 & 847933 \\
\hline 5 & 10 & 5086451 & 847086 \\
\hline \multicolumn{3}{|c|}{} & 847147 \\
\hline Mean of RR & 457.2608 \\
\hline S.D of RR & 0.053976 \\
\hline
\end{tabular}

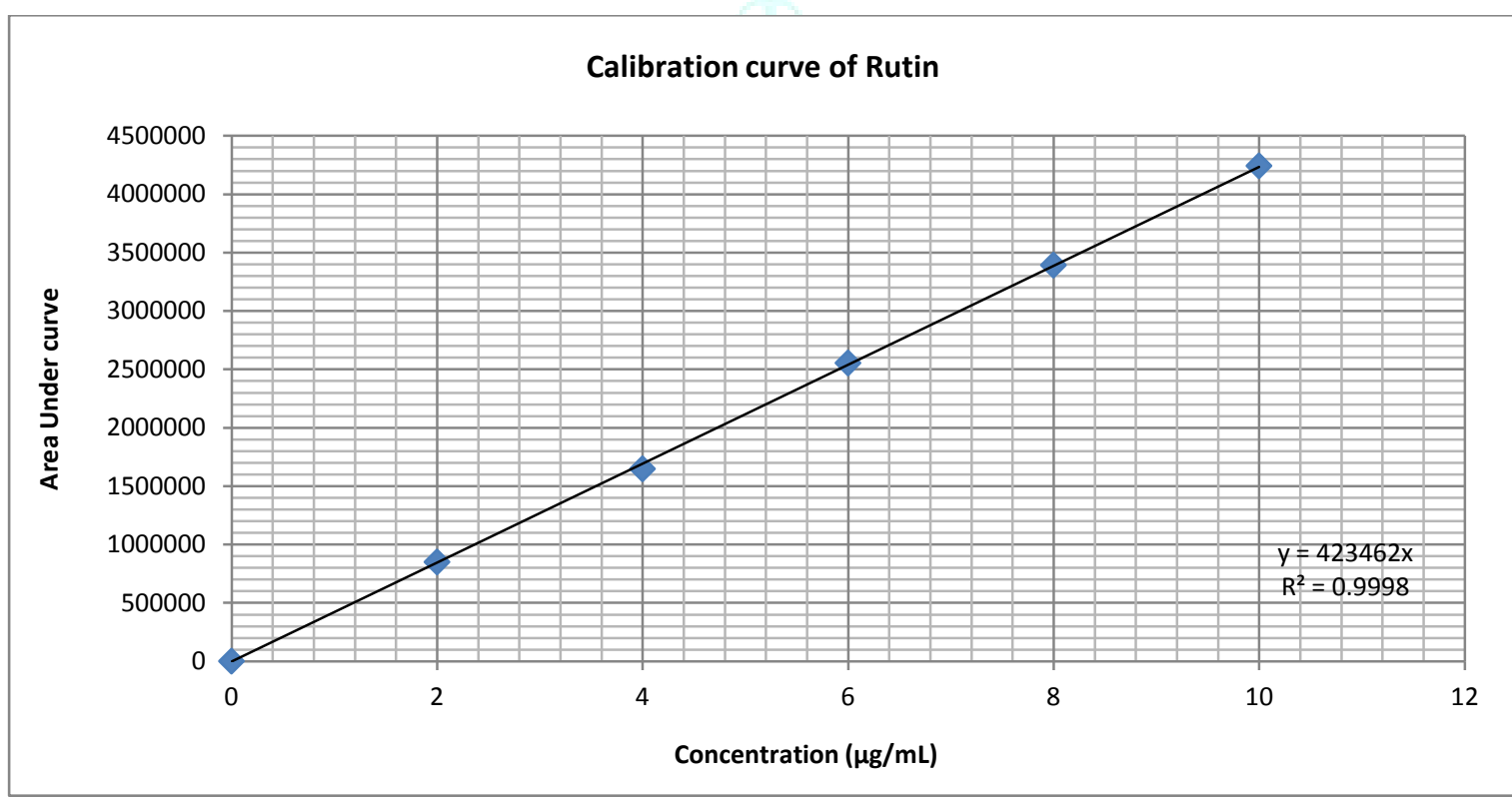

Figure 6: Calibration curve of Rutin

\subsection{Precision of the developed method}

Repeatability (Intra-day precision) was studied by calculating the relative standard deviation (RSD) for nine replicates of the concentration of $10 \mu \mathrm{g} / \mathrm{mL}$, performed on the same day and under same experimental conditions. The results of rutin determinations in the working standard solution with the relative standard deviation were calculated (Table 3). Inter-day precision studies include the estimation of variations in analysis when a method is used within laboratories, on different day. The RSD values obtained for intraday \& inter-day precision rutin were $0.03527 \& 0.026154906$ respectively. 
Table 3 Precision data

\begin{tabular}{|c|c|c|c|}
\hline S. No. & Concentration $(\boldsymbol{\mu g} / \mathbf{m L})$ & $\begin{array}{c}\text { AUC } \\
\text { (Same day) }\end{array}$ & $\begin{array}{c}\text { AUC } \\
\text { (Different day) }\end{array}$ \\
\hline 1 & 10 & 4243588 & 4240725 \\
\hline 2 & 10 & 4239365 & 4240440 \\
\hline 3 & 10 & 4243140 & 4241232 \\
\hline 4 & 10 & 4241572 & 4241426 \\
\hline 5 & 10 & 4242064 & 4241995 \\
\hline 6 & 10 & 4240228 & 4242352 \\
\hline 7 & 10 & 4241934 & 4243728 \\
\hline 8 & 10 & 4242782 & 4242506 \\
\hline 9 & 10 & 4239884 & 4243232 \\
\hline & Mean of AUC & 4241617.444 & 4241959.556 \\
\hline \multicolumn{2}{r|}{ S.D. of AUC } & 1494.491143 & 1109.480521 \\
\hline & \% R.S.D of RR & 0.035233992 & 0.026154906 \\
\hline
\end{tabular}

\subsection{Robustness of the developed method}

The robustness of the proposed method was evaluated by deliberately changing the chromatographic conditions such as flow rate, solvent ratio and $\mathrm{pH}$. The results showed that varying the chromatographic conditions had no appreciable effects on the chromatographic parameters (Table 4)

Table 4 Robustness study of the proposed HPLC method

\begin{tabular}{|c|c|c|}
\hline Parameter & Conditions & Retention Time \\
\hline \multirow{3}{*}{ Flow Rate (mL/min } & 0.9 & 6.1 \\
\cline { 2 - 3 } & 1 & 5.71 \\
\cline { 2 - 3 } & 1.1 & 5.25 \\
\hline \multirow{3}{*}{ Mobile Ratio (v/v) } & $75: 25$ & 6.15 \\
\cline { 2 - 3 } & $80: 20$ & 5.71 \\
\cline { 2 - 3 } & $85: 15$ & 5.32 \\
\hline \multirow{3}{*}{$\mathrm{pH}$} & 3.1 & 5.9 \\
\cline { 2 - 3 } & 3.2 & 5.71 \\
\cline { 2 - 3 } & 3.3 & 5.35 \\
\hline
\end{tabular}

Table 5: System suitability studies for estimation of rutin by HPLC

\begin{tabular}{|c|l|c|}
\hline S. No. & \multicolumn{1}{|c|}{ Parameters } & Inference \\
\hline 1 & Linearity range & $2 \mu \mathrm{g} / \mathrm{mL}-10 \mu \mathrm{g} / \mathrm{mL}$ \\
\hline 2 & Regression equation & $\mathrm{y}=42346 \mathrm{x}$ \\
\hline 3 & Correlation coefficient & 0.999 \\
\hline 4 & Asymmetric factor & 1.948 \\
\hline 5 & Tailing factor & 1.277 \\
\hline 6 & Theoretical plates & 7134.155 \\
\hline 7 & Resolution & 6.457 \\
\hline 8 & LOD $(\mathrm{ng} / \mathrm{mL})$ & $100 \mathrm{ng} / \mathrm{mL}$ \\
\hline 9 & LOQ $(\mathrm{ng} / \mathrm{mL})$ & $\mathrm{ng} / \mathrm{mL}$ \\
\hline
\end{tabular}

\subsection{Limit of detection and quantification}

LOD \& LOQ were calculated by using the following equations.

$$
\begin{aligned}
& L O D=3.3 X \frac{\mathrm{SD}}{\mathrm{S}} \text { And } \\
& L O Q=10 X \frac{\mathrm{SD}}{\mathrm{S}}
\end{aligned}
$$

Where SD = the standard deviation of the response,

$\mathrm{S}=$ Slope of the calibration curve.

The LOD value was found to be $100 \mathrm{ng} / \mathrm{mL}$ and the LOQ value was found to be $300 \mathrm{ng} / \mathrm{mL}$ for the rutin.

\section{CONCLUSIONS}

The proposed analytical method for estimation of rutin in the extracts of Prosopis cineraria is accurate, precise, linear, robust, reproducible and within the range. The results show that Prosopis cineraria contain considerable amounts of flavonoids which demonstrate that the plant could be considered as a potential source of natural healthpromoting antioxidants for medicinal and food applications. This study established a quantitative method for the determination of rutin from Prosopis cineraria.

Acknowledgments: Authors are thankful to head of department, SOPS UTD RGPV Bhopal for providing required facilities for partial research work at SAIL SOPS. 


\section{REFERENCES}

1. Liu Y, Singh D, Nair M, Pods of Khejri (Prosopis cineraria) consumed as a vegetable showed functional food properties. Journal of Functional Foods, 2012; 4(1):116-121. doi: $10.1016 /$ j.jff.2011.08.006

2. Anand S, Thakur S, Gargi M, Choudhary S, Bhardwaj P, Development and characterization of genomic microsatellite markers in Prosopis cineraria. Current Plant Biology, 2017; 910:37-42. doi: 10.1016/j.cpb.2017.03.001

3. Garg A, Mittal S, Review on Prosopis cineraria: A potential herb of Thar desert. Drug Invention Today, 2013; 5(1):60-65. doi: 10.1016/j.dit.2013.03.002

4. Ross J, Kasum C, Dietary Flavonoids: Bioavailability, Metabolic Effects, and Safety. Annual Review of Nutrition, 2002; 22(1):19-34

doi: 10.1146/annurev.nutr.22.111401.144957

5. Cody V, ChemInform Abstract: Crystal and Molecular Structures of Flavonoids. Cheminform, 1989; 20(12). doi: 10.1002/chin.198912363

6. Cao J, Zhang Y, Chen W, Zhao X, The relationship between fasting plasma concentrations of selected flavonoids and their ordinary dietary intake. British Journal Of Nutrition, 2009; 103(02):249. doi: 10.1017/s000711450999170x

7. Hakkinen S, Kärenlampi S, Heinonen I, Mykkanen $\mathrm{H}$, Torronen A, Content of the Flavonols Quercetin, Myricetin, and Kaempferol in 25 Edible Berries. Journal of Agricultural And Food Chemistry, 1999; 47(6):2274-2279. doi: 10.1021/jf9811065

8. Scholz, Williamson, Interactions Affecting the Bioavailability of Dietary Polyphenols in Vivo. International Journal For
Vitamin And Nutrition Research, 2007; 77(3):224-235. doi: 10.1024/0300-9831.77.3.224

9. Manach C, Williamson G, Morand C, Scalbert A, Rémésy C Bioavailability and bioefficacy of polyphenols in humans. I. Review of 97 bioavailability studies. The American Journal of Clinical Nutrition, 2005; 81(1):230S-242S. doi: 10.1093/ajcn/81.1.230s

10. Shah W, Rane N, Kekare MB, Vaidya V, Estimation of two bioactive compounds from Azadiracta indica A.Juss. leaves using HPLC. International Journal of Pharma and Bio Sciences; 2010; 1(2):1-7.

11. Behnaz M, Davood E A, Atena A, Simultaneous determination of rutin and quercetin in different parts of Capparis spinosa, Bulletin of environment, Pharmacology and Life Sciences 2013; 2(2):35-38.

12. Crozier, A, Jensen E, Lean M, McDonald M, Quantitative analysis of flavonoids by reversed-phase high-performance liquid chromatography. Journal Of Chromatography A, 1997; 761(1-2):315-321. doi: 10.1016/s0021-9673(96)00826-6

13. Wang S, Huang K, Determination of flavonoids by highperformance liquid chromatography and capillary electrophoresis. Journal of Chromatography A, 2004; 1032(12):273-279. doi: 10.1016/j.chroma.2003.11.099

14. Ashok Kumar BS, Lakshman K, Jayaveera KN, Vamshi Krishna $\mathrm{N}$, Manjunath M, Suresh MV, Estimation of rutin and quercetin in Amaranthus viridis Linn by HPLC, Asian Journal of Experimental Sciences, 2009; 23:51-54.

15. ICH, Q2, Text on validation of Analytical procedures: Methodology, International conference on Harmonization, 2005. 